\title{
Resource Reservation and Allocation Method for Next Generation Mobile Communication Systems
}

\author{
Jongchan Lee ${ }^{1}$, Sok-Pal Cho ${ }^{2}$, and Chiwon Kang ${ }^{3}$ \\ ${ }^{1}$ Senior Researcher, Mobile Access Research Team, ETRI, Korea \\ chan2000@etri.re.kr \\ ${ }^{2}$ Dept. of C\&C. Eng. Sungkyul Univ., Korea \\ ${ }^{3}$ Senior Researcher, KDM, Korea
}

\begin{abstract}
This paper proposes a handoff scheme to transmit multimedia traffic based on the resource reservation procedure using direction estimation. The handoff requests for real-time sessions are handled based on the direction prediction and the resource reservation scheme. In simulation results, proposed method provides a better performance than the previous method.
\end{abstract}

\section{Introduction}

As mobile users move around, the network must continuously track them down and discover their new locations in order to be able to deliver data to them. Especially wireless resources availability varies frequently as users move from one access point to another $[1,2]$. In order to deterministically guarantee QoS support for a mobile, the network must have prior exact knowledge of the mobile's path.

Majority of the previous schemes to support mobility make a reservation for resources in adjacent cells [3, 4]. The reserved resource approach offers a generic means of improving the probability of successful handoffs by simply reserving the corresponding resources exclusively for handoff sessions in each cell. The penalty is the reduction in the total carried traffic load due to the fact that fewer resources are granted to new sessions.

\section{Proposed Structure}

The base station reserves only the resources corresponding to the minimum transmission rate to the mobile. Based on the location and the direction of the mobile within a cell, the resource reservation is performed with the following order: unnecessary state, not necessary state, necessary state, and positively necessary state. If the reservation variable for the mobile is changed, the reservation is canceled and the resources have to be released with the reverse order and returned to the fool of available resource. The set of the reserved resources have its priorities depending on whether it can be 
allocated to new sessions or not: a real-time handoff session (priority 1), a non-realtime handoff session (priority 2) and a non-real-time new session (priority 3). This strategy is explained in the following thing.

\section{Resource Reservation ( ) \\ while \\ If (Unnecessary State) then}

The resource reservation needs not be performed;

else if (Not Necessary State) then

if (there are available resources in each of the estimated cells) then

Reserve the resources;

\section{end if}

If (enough resources are not available for a new session in the estimated cells) then

The reserved resources is occupied by the new sessions;

end if

else if (Necessary State) then

if (no resources are available for the reservation in the estimate cell) then

Allocate and reserve the shared resources for a real-time session;

end if

If (there is no enough resource available to accommodate a new session in the estimated cells) then

The reserved resources for real-time handoff sessions can be occupied by non-real-time new sessions;

end if

else if (Positively Necessary State) then

if (no resources are available for the reservation in the estimate cell) then

Allocate and reserve the shared resources for a real-time sessions and non-real-time sessions;

end if

If (there is no enough resource available to accommodate a new session in the estimated cells) then

New sessions cannot occupy the reserved resources;

end if

end if

Resource Allocation ()

while

If (handoff session) then

if (Real-time class) then

if (there is reserved resource) then

Admit the handoff session;

Allocate the reserved resource;

else if (there is available resource) then

Admit the handoff session 


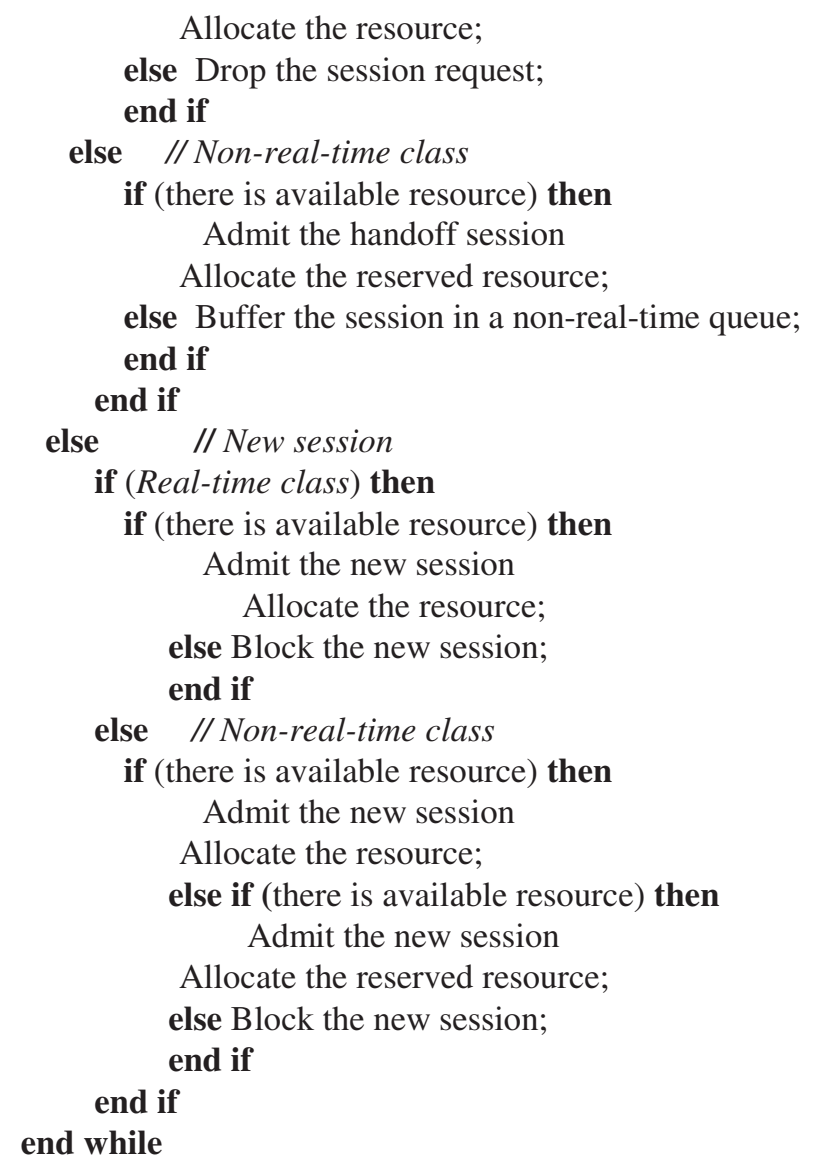

\section{Performance Analysis}

The simulation model is based on a B3G system proposed from ETRI, which is implemented using MOBILESimulatorV5. The simulation model composed of a single cell, which will keep contact with its six neighboring cells. Each cell contains a base station, which is responsible for the session setup and tear-down of new applications and to serve handoff applications. The moving path and the mobile velocity are affected by the road topology. The moving pattern is described by the changes in moving direction and velocity. Fig. 1 and Fig. 2 shows the variation in the dropping rates in the different strategies when arrival rate of new session requests is increased. Results demonstrate that the dropping rate of the direction-based has decreased to about $20 \%$ and $15 \%$ for real-time and non-real-time sessions, as compared to the Fixedbased and dynamic-based, respectively. Handoff dropping rate for the dynamic scheme is much better than that for Fixed. 


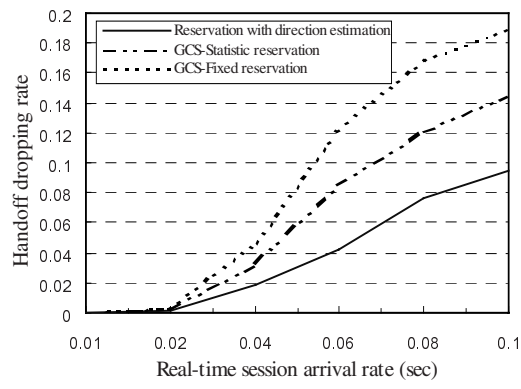

Fig. 1. Real-time sessions

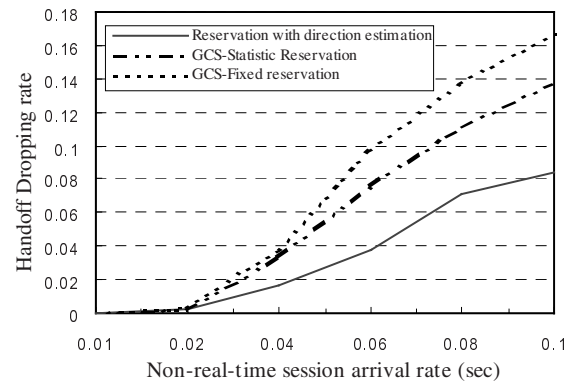

Fig. 2. Non-real-time sessions

\section{Conclusions}

This paper goal is to address the problem of guaranteeing an acceptable level of QoS requirements for mobile users as they move from one location to another. This is achieved through reservation variables such as the current location and the moving direction that is presented with a set of attributes that describes the user mobility. Based on reservation variables a scheme that provides predictive QoS guarantees in mobile multimedia networks is proposed. We have focused in improving the overall system performance.

\section{References}

1. Ab. Aljadhai, Taieb F. Znati, "Predictive Mobility Support for QoS Provisioning in Mobile Wireless Environments," IEEE JSAC., Vol. 19, No. 10, Oct., 2001.

2. W. C. Y. Lee, "Smaller cells for greater performance," IEEE Com. Mag., 1999.

3. O. T. W. Yu and V. C. M. Leung, "Adaptive Resource Allocation for Prioritized Call Admission over an ATM-based Wireless PCN," IEEE JSAC., Vol. 15, pp. 1208-1225, Sept. 1997.

4. L. Ortigoza-Guerrero and A. H. Aghvami, "A Prioritized Handoff Dynamic Channel Allocation Strategy for PCS,” IEEE Trans. Vehic.Tech., vol. 48, No. 4, pp. 1203-1215, Jul. 1999. 